\title{
Angle-Scanning Surface Plasmon Resonance System with 3D Printed Components for Biorecognition Investigation
}

\author{
ChenGuang Zhang, ${ }^{1}$ Ching-Jung Chen ${ }^{D},{ }^{1,2}$ Kalpana Settu, ${ }^{3}$ and Jen-Tsai Liu ${ }^{4}{ }^{4}$ \\ ${ }^{1}$ School of Electronic, Electrical and Communication Engineering, University of Chinese Academy of Sciences, China \\ ${ }^{2}$ School of Opto-Electronic Technology, University of Chinese Academy of Sciences, China \\ ${ }^{3}$ Department of Electrical Engineering, National Taipei University, Sanxia, Taiwan \\ ${ }^{4}$ College of Materials Science and Opto-Electronic Technology, University of Chinese Academy of Sciences, China
}

Correspondence should be addressed to Ching-Jung Chen; cjchen@ucas.ac.cn and Jen-Tsai Liu; jtliu@ucas.ac.cn

Received 7 April 2018; Revised 17 May 2018; Accepted 24 May 2018; Published 11 July 2018

Academic Editor: Shuan-Yu Huang

Copyright (c) 2018 ChenGuang Zhang et al. This is an open access article distributed under the Creative Commons Attribution License, which permits unrestricted use, distribution, and reproduction in any medium, provided the original work is properly cited.

\begin{abstract}
Surface plasmon resonance (SPR) is a real-time, label-free, and high-sensitive detection technology. SPR has been widely used in many applications such as biomolecular interaction analysis, environmental monitoring, and medical diagnostics. However, conventional SPR sensor systems usually require expensive equipment and complicated optics. In this paper, we have demonstrated a rapid prototyping of angle-scanning SPR for bioanalytical investigation. Rapid prototyping was attained by utilizing the FDM (fused deposition modeling) based 3D (three-dimensional) printing technology. Two rotating platforms were employed to drive the laser source and photodiode, respectively. A temperature regulation unit was incorporated to maintain the system temperature in order to reduce the temperature effect. The proposed SPR rapid prototyping yielded a refractive index resolution of $6.4 \times 10^{-6}$ RIU (refractive index unit), and the biotin-avidin system validated the kinetics parameters measurement capability. The obtained results indicated that the FDM 3D printing has great potential for developing rapid-prototyping SPR system.
\end{abstract}

\section{Introduction}

Surface plasmon resonance (SPR) is a surface-sensitive optical technique that is used to study a thin layer on a metal surface. When P-polarized light is projected onto metal film, it can excite the oscillation of the electrons on the surface of the film. This oscillation is called surface plasmon wave (SPW). When the wave vector of the incident light matches the wave vector of SPW, the reflected light intensity decays rapidly, and the corresponding angle of the incident light is called resonance angle. Surface plasmon resonance (SPR) is a powerful analytical technology. It can detect the thickness of the films absorbed onto the sensor surface and interactions between biomolecules such as antigen-antibody or protein-DNA [15]. In comparison with traditional detection methods, like X-ray, HPLC, and HPLC-MS, SPR technology can perform kinetics parameters (association and dissociation process) measurement, concentration measurement, and real-time molecule detection. It has been widely used in chemical, biology, agriculture, environment, and food safety [6-12].

Traditional SPR systems usually require expensive equipment and complicated optics. The cost of commercial SPRs varies from $\$ 10,000$ to $\$ 5,000,000$, and the refractive index resolution ranges from $10^{-5}$ RIU (refractive index unit) to $10^{-7}$ RIU. Many optical-fiber based SPRs have been studied intensively in the literature [13-15]. The structure of these devices is relatively simple. However, the resolution and dynamic range are often sacrificed $[16,17]$. To reach a high resolution of $10^{-6} \mathrm{RIU}$ or $10^{-7} \mathrm{RIU}$, a spectrometer is always required for an optical-fiber SPR [18].

Compared with optical-fiber SPR, angle-scanning SPR has the capability of achieving high resolution and wide refractive index measurement range. A specific wavelength laser is irradiated on a metal film surface and scanned to achieve certain range of incident angle. However, anglescanning SPR requires more mechanical components, and 
also the optical path is more complicated. Typical anglescanning SPRs are based on Kretschmann configuration. It involves many mechanical components manufacturing and complex optical path design. Therefore, constructing a compact home-made SPR is of great importance.

3D printing, also called additive manufacturing, since its origins in 1980s has been a tool for developing rapidprototyping products. With the expiration of $3 \mathrm{D}$ printing technology patents, this field has witnessed a great growth [19]. FDM (fused deposition modeling) is one of the most popular $3 \mathrm{D}$ printing technologies. In FDM 3D printing, a heated nozzle is employed to melt raw material and extrude fused material onto a building platform, and it lays the fused material in layers. A wide range of materials can be printed, such as acrylonitrile butadiene styrene (ABS), polylactic acid (PLA), high-impact polystyrene (HIPS), and thermoplastic polyurethane (TPU). After the emergence of open source RepRap project, desktop fused deposition modeling (FDM) $3 \mathrm{D}$ printers have become more popular among users, manufacturers, and researchers. It is simple, cost effective, and versatile. Application of FDM 3D printing technology to toys, prototypes, scientific tools, and medical equipment becomes more and more common [19-23].

Using $3 \mathrm{D}$ printing technology to design an SPR is an interesting and attractive thing. Recently, some studies have demonstrated the use of 3D printing in SPR platform. Hasan et al. developed a smartphone based SPR imaging platform for on-site biodetection [16]. They utilized the 3D printing technique just to print the device holder. This system has a dynamic range less than 0.02 RIU, which limits the application for measuring large refractive index change. Bonyár et al. proposed an acrylic photopolymer material-based 3D printing to fabricate molds for PDMS (polydimethylsiloxane) casting and flow cell for SPR instrument. This method enabled the customization of SPR flow cell alone [24]. However, 3D printing technology could be further applied to SPR. The use of $3 \mathrm{D}$ printing technology simplifies the process of manufacturing, accelerates the design of prototype, and makes designs more flexible. Furthermore, complex equipment, such as lathes and milling machines, is not important any more, even nonexpert users can quickly copy and build a $3 \mathrm{D}$ printed project.

In this study, we have demonstrated a rapid-prototyping angle-scanning SPR utilizing FDM 3D printing technology. PLA filament was chosen for its relative high tensile strength $(\sim 50 \mathrm{MPa})$, good reproducibility, and low cost. With the help of $3 \mathrm{D}$ printing, the flow cell and optical path were designed to be modular and customizable. Refractive index resolution was found to be $6.4 \times 10^{-6}$ RIU. To validate the bioanalytical capability, biotin-avidin system was used as target analyte.

\section{Method}

Biotin and Avidin were purchased from Macklin Biochemical Co. Ltd. (Shanghai, China). N-hydroxysulfosuccinimide sodium salt (sulfo-NHS) and 1-(3-dimethylaminopropyl)3-ethylcarbodiimide hydrochloride (EDC) were purchased from Aladdin Chemistry Co. Ltd. (Shanghai, China). Cysteamine and Sodium dodecyl sulfate (SDS) were obtained from Beijing Biotopped Science \& Technology Co. Ltd. (Beijing, China). Absolute ethyl alcohol and $\mathrm{NaCl}$ were purchased from Beijing Chemical Works (Beijing, China). Deionized water obtained from a CCT-3300 water purification system was used in all the experiments. Matching oil series E (1.520) was purchased from Cargill Dow LLC. (USA). Polylactide (PLA) 3D printing filaments $(1.75 \mathrm{~mm})$ were purchased from 3D Systems (USA).

The developed SPR system includes a $650 \mathrm{~nm}$ laser source (Shenzhen Fuzhe Technology Co. Ltd. 150mW, Shenzhen, China), a photodiode (2DU $10 \mathrm{~mm} \times 10 \mathrm{~mm}$ ), a polarizer $(\mathrm{F}-\mathrm{PZP}-001 \phi 15 \mathrm{~mm} \times 2 \mathrm{~mm})$, and a BK7 triangle prism $(20 \mathrm{~mm} \times 20 \mathrm{~mm} \times 10 \mathrm{~mm})$. Au-coated glass slides $(2 \mathrm{~mm} \times$ $8 \mathrm{~mm} \times 30 \mathrm{~mm}$ ) were prepared by coating $47.5 \mathrm{~nm}$ thickness of Au layer using sputtering deposition method. Prior to the deposition the $\mathrm{Au}$ film, the glass slide was precoated with $2 \mathrm{~nm}$ chromium layer to enhance the adhesion of the $\mathrm{Au}$ film. Cube 3 3D printer (3D Systems, USA) was used to print the necessary SPR system components (Fig S1). Silhouette Portrait (Silhouette America, Inc.) was used to cut the flow cell. Two rotating platforms (Beijing PDV Instrument Co., Ltd., China) were employed as the mechanical drivers. Open source boards (Arduino Mega 2560) accompanied with a self-made amplifier circuit comprise the hardware circuits. Two TECs (Thermoelectric Coolers) TES1-12704 (Hebei I.T. Co. Ltd., $3.3 \mathrm{~mm} \times 30 \mathrm{~mm} \times 30 \mathrm{~mm}$, Shanghai, China) were adapted as the temperature controller.

Schematic of rapid-prototyping angle-scanning SPR system block diagram is shown in Figure 1. Light from the laser source was first $\mathrm{p}$-polarized by a polarizer and then projected onto the sensor surface via a triangle prism. The reflected light from the sensor surface was collected by a photodiode. The collected raw signal was amplified by a self-made amplifier circuit and sampled the built-in ADC (analog to digital converter) of the microcontroller unit ATmega2560 (Microchip Technology Inc., 8-bit AVR microcontroller, Chandler, Arizona, USA). Digital signals were eventually transmitted to the software on computer for analysis. The platform controller can drive the system to scan from $40^{\circ}$ to $72^{\circ}$. When performing measurements, instead of scanning a whole SPR spectrum, an angle range of $3^{\circ}$ around the resonance angle was scanned at a speed of $0.3^{\circ} / \mathrm{s}$. For the temperature regulation unit, low temperature drift coefficient NTC (Negative Temperature Coefficient) resistors (SEMITEC Corporation, 103AT-4 Shape1, Japan) were employed to sense the system temperature and signals from NTC resistors were quantified by a 16-bit high-precision ADC ADS1115 (Texas Instruments Inc. USA). A microcontroller ATmega328 (Microchip Technology Inc., 8-bit AVR microcontroller, Chandler, Arizona, USA) was used to obtain the temperature information from ADS1115 via IIC (Inter-Integrated Circuit) communication protocol and control the heating or cooling of the flow cell through TECs. A PID (proportional-integral-derivative) controller algorithm was incorporated with ATmega328 to maintain a stable temperature circumstance.

The flow cell module consists of rubber flow cell and stainless part. Arrangement of the flow cell module is shown in Fig. S2. The rubber flow cell of volume $65 \mu \mathrm{L}$ and dimension $5.3 \mathrm{~mm} \times 23 \mathrm{~mm} \times 0.5 \mathrm{~mm}$ was designed and constructed 


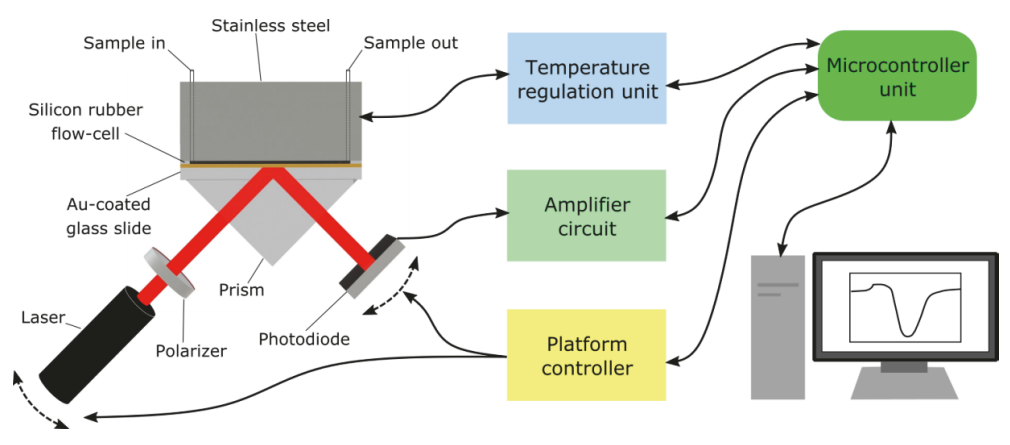

FIGURE 1: Schematic representation of the complete SPR system.

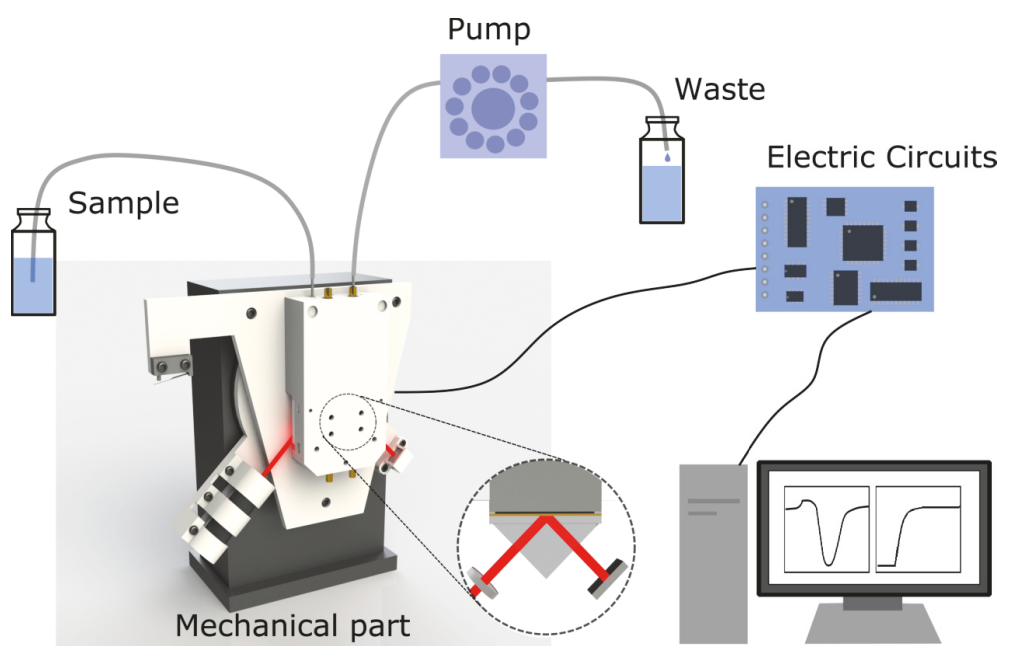

FIGURE 2: Overview of the designed SPR system by rapid-prototyping 3D printing.

using desktop cutter Silhouette Portrait. However, one can customize the shape of flow cell by modifying the digital design file and snip it in the rubber film with Silhouette Portrait.

Overview of the developed rapid-prototyping SPR system was depicted in Figure 2. The system was constructed with $3 \mathrm{D}$ printed components. Cube 3 desktop version $3 \mathrm{D}$ printer was used to print the components with PLA filaments. The component models were designed using SolidWorks 2012 software and saved as STL (Standard Triangle Language) file format. Then, these STL files were imported into the $3 \mathrm{D}$ printer's software Cubify version 3.9.0 (3D systems, USA). Two rotating platforms were employed and a $3 \mathrm{D}$ printed optical platform was installed onto the rotating platforms. The laser source and photodiode can be easily mounted onto the optical platform with the $3 \mathrm{D}$ printed holders. Unlike the laser and photodiode, the flow cell module was removable and customizable. Furthermore, the alignment of optical parts can be achieved effortlessly.

The SPR sensor functionalization process is shown in Figure 3. To functionalize the SPR sensor with biotin, Aucoated glass slide was first cleaned ultrasonically with $4 \%$ sodium dodecyl sulfate (SDS) solution and rinsed with deionized water. Then it was exposed to UV light for 20 minutes. Au-coated glass slide was then immersed in $10 \mathrm{mM}$ cysteamine solution for 2 hours and subsequently rinsed with deionized water to remove the residual cysteamine. The resulting sensor was then immersed in $0.2 \mathrm{mg} / \mathrm{mL}$ biotin solution containing $15 \mathrm{mM}$ EDC and $5 \mathrm{mM}$ sulfo-NHS. Then the sensor was rinsed in deionized water and absolute ethanol and dried under a stream of nitrogen for further use.

\section{Experimental}

We used Tianmin contact angle meter to measure water contact angle at each modification stage. SPR sensor chips were mounted on the plane of contact angle meter. $4 \mu \mathrm{L}$ of deionized water was dropped onto the sensor chip surface. The droplet cross-section was recorded as image file by the camera on the contact angle meter. Open source software ImageJ was used to estimate the contact angle from recorded image files.

The most benefit of SPR biosensor technology is the determination of kinetics of biomolecular interactions. It can be determined that the interaction of analytic A and ligand B is immobilized on sensor surface. It usually uses the simplest case $\mathrm{A}+\mathrm{B} \longrightarrow \mathrm{AB}$. In this study, The kinetic parameters measurement capability of the developed system was validated using the biotin-avidin system. Affinity interactions between biotin and avidin were characterized by the association rate constant $k_{a}$, dissociation rate constant $k_{d}$, and equilibrium 


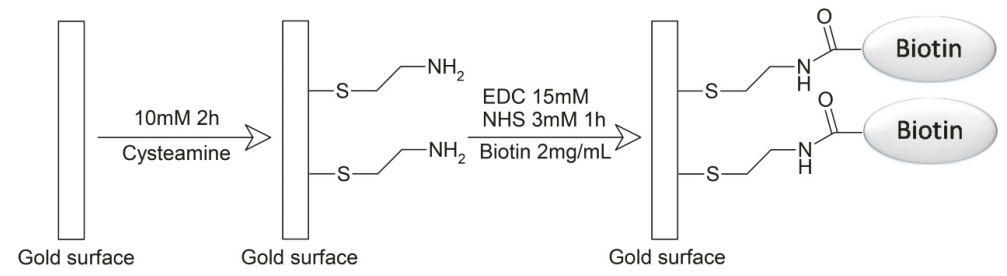

FIGURE 3: SPR sensor modification process.

association constant $K_{e q}$, where $\mathrm{A}$ is the injected analyte, $\mathrm{B}$ is the immobilized ligand, and $\mathrm{AB}$ is the analyte-ligand complex. The experiment data was fitted with $\mathrm{A}+\mathrm{B} \longrightarrow \mathrm{AB}$ interaction model. The interaction process can be described as (1). The response signal $R$ of SPR system is proportional to the amount of $[\mathrm{AB}], k_{a p p}$ is the apparent reaction rate constant, $t$ is the interaction time, and the maximum response signal $R_{\max }$ is proportional to the initial $[B]$. A series of concentrations of $[A]$ solutions were used to derive the relationship between $k_{a p p}$ and different concentrations of $[A]$, and a regression curve was obtained from (2). Thus $k_{a}$ is the slope of the regression, $k_{d}$ is the intercept of the regression curve, and $K_{e q}$ is computed as $k_{a} / k_{d}$.

$$
\begin{gathered}
R=\frac{k_{a}[A] R_{\max }}{\left([A] k_{a}+k_{d}\right)}\left[1-e^{-k_{a p p} t}\right] \quad k_{a p p}=[A] k_{a}+k_{d} \\
k_{a p p 1}=\left[A_{1}\right] k_{a}+k_{d} \\
k_{a p p 2}=\left[A_{2}\right] k_{a}+k_{d} \\
\ldots \\
k_{a p p n}=\left[A_{n}\right] k_{a}+k_{d}
\end{gathered}
$$

For fast kinetics parameters measurement, affinity interactions between biotin and avidin were characterized by the association rate constant $k_{a}$, the dissociation rate constant $k_{d}$, and the equilibrium association constant $K_{e q}$. However, the interaction process is divided into association phase, equilibrium phase, and dissociation phase. The dissociation rate constant $k_{d}$ was obtained from the dissociation phase by (3). Then it was substituted into (1) to obtain $k_{a}$ from the association phase. Thus $K_{e q}$ was computed as the ratio of association and dissociation rate constants.

$$
\frac{d R}{d t}=-k_{d} R
$$

\section{Results and Discussion}

Prior to conducting the biomedical experiment, effectiveness of the sensor surface modification should be verified. Contact angle measurement is a powerful tool to examine every step during the sensor functionalization. Results of contact angle measurement are shown in Figure 4. Bare Au surface exhibited a value of $88.378^{\circ}$. After $20 \mathrm{~min}$ of UV exposure, the contact angle decreased to $71.922^{\circ}$. This contact decrease indicates that the UV exposure could effectively remove the impurities on the Au film. When the cysteamine self-assembled monolayers (SAMs) formed on $\mathrm{Au}$ film, the contact angle became $46.720^{\circ}$, which indicated that the cysteamine SAM is terminated with a hydrophilic domain $-\mathrm{NH}_{2}$ [25]. Subsequently, after the immobilization of biotin onto the cysteamine SAM, the contact angle decreased to $21.911^{\circ}$ from $46.720^{\circ}$ of the cysteamine SAM. The reason was that the ureido- and tetrahydrothiophene-rings of biotin are more hydrophilic than $-\mathrm{NH}_{2}$ terminated cysteamine SAM [26]. The contact angle of biotin SAM was smaller than the previously reported one in study [27], and this may be due to the well-organized biotin SAM. Thus, the above results showed that the SPR sensor chip was prepared successfully.

The interactions between biotin and different concentrations of avidin solution are plotted in Figure 5. A higher concentration of avidin showed a faster association rate. Biotin SAM on the sensor surface was saturated in a short time. Meanwhile, at lower concentration of avidin, the association process occurred slowly, and it took a longer time to reach equilibrium. After the saturation of biotin on the sensor surface, the binding of biotin and avidin reaches equilibrium, and the SPR response signal reaches $R_{\max }$. The relationship between $k_{a p p}$ and various concentrations of avidin was plotted as shown in Figure 6. The linear regression equation was obtained as $k_{a p p}=4.9 \times 10^{4} \times[C]+5.4 \times 10^{-5}$, where $\mathrm{C}$ is the avidin concentration. Thus the kinetic parameter $k_{d}$ was first obtained as $5.4 \times 10^{-5} \mathrm{~s}^{-1}, k_{a}$ was obtained as $4.9 \times 10^{4} \mathrm{M}^{-1} \mathrm{~s}^{-1}$, and the $K_{e q}$ was computed as $9.8 \times 10^{8} \mathrm{M}^{-1}$, which is close to the $K_{e q}$ reported by Zhao et al. [28].

For fast kinetic parameters measurement, $73.2 \mathrm{nM}$ avidin was used. The interaction process during the association phase, equilibrium phase, and dissociation phase was recorded as in Figure 7. For the dissociation phase, $k_{d}$ was derived as $0.0012 \mathrm{~s}^{-1}$. After substituting $k_{d}$ into (1), we obtain $k_{a}=3.551 \times 10^{4} \mathrm{M}^{-1} \mathrm{~s}^{-1}$ and $K_{e q}=k_{a} / k_{d}=2.96 \times 10^{7} \mathrm{M}^{-1}$. The kinetics parameters obtained using the two above methods were different. However, the fast kinetics parameters measurement utilizes the association and dissociation phase of the interaction process at only one concentration. Comparing these two methods, the results showed that the system has the capability to analyse the interaction between protein and ligands by different analytic concentrations.

\section{Conclusions}

This work presented the development of a rapid-prototyping surface plasmon resonance utilizing FDM 3D printing 


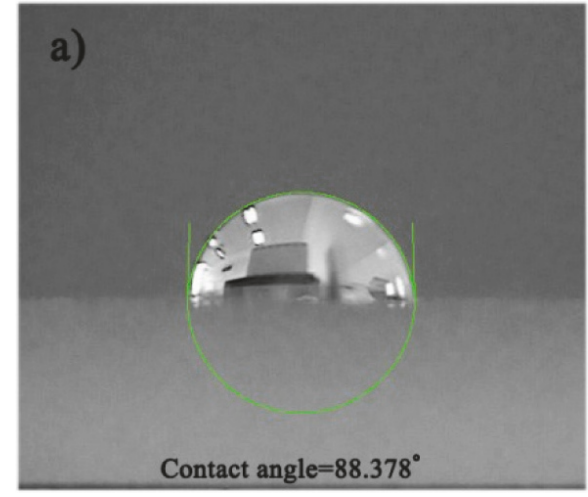

(a)

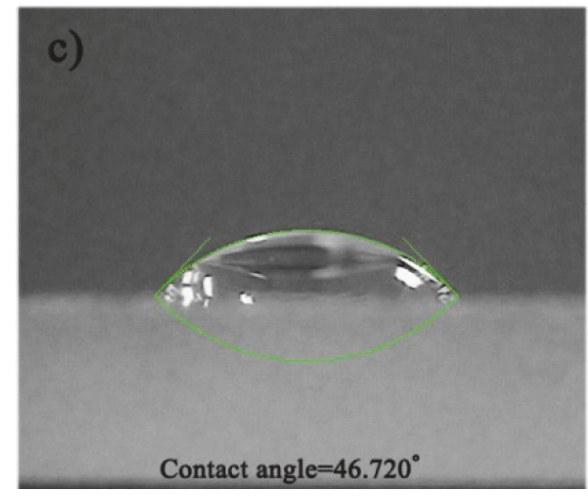

(c)

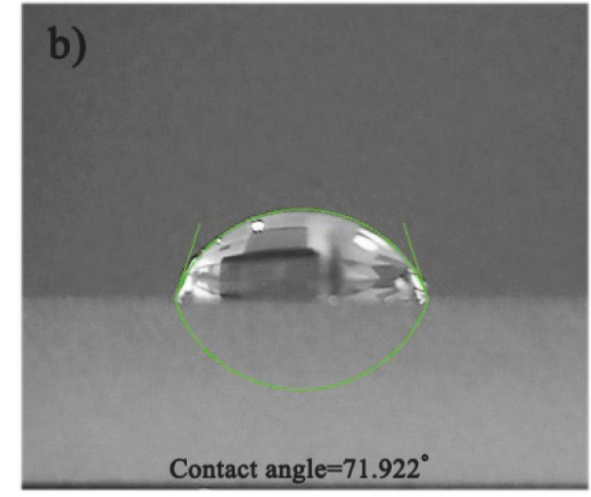

(b)

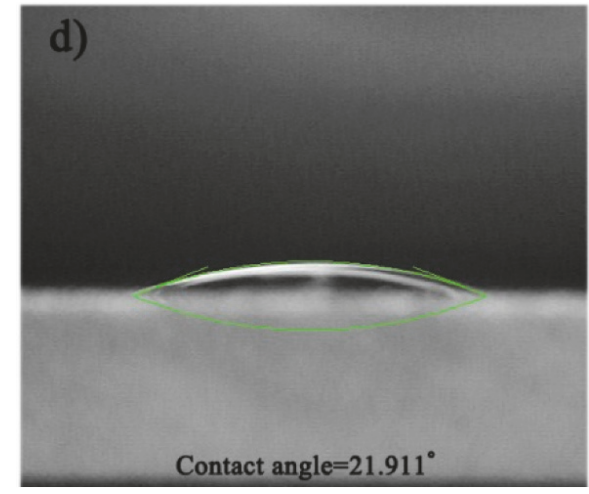

(d)

Figure 4: Contact angle measurement: (a) bare Au film; (b) Au film after 20 min UV exposure; (c) Au/cysteamine SAM; (d) Au/cysteamine/ biotin film.

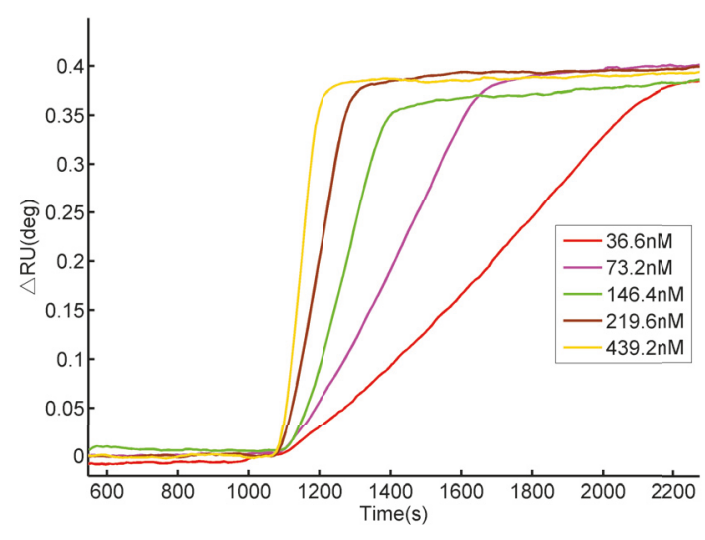

FIGURe 5: Association curve of biotin and avidin.

technology. The developed device was modular and the optical path and flow cell were customizable. The system performance was validated with a series of experiments, and our system showed a high resolution of $6.4 \times 10^{-6}$ RIU. Furthermore, kinetics parameters measurement capability was validated by biotin-avidin system. In the future, we will aim to develop our 3D-SPR to be more modular and easier

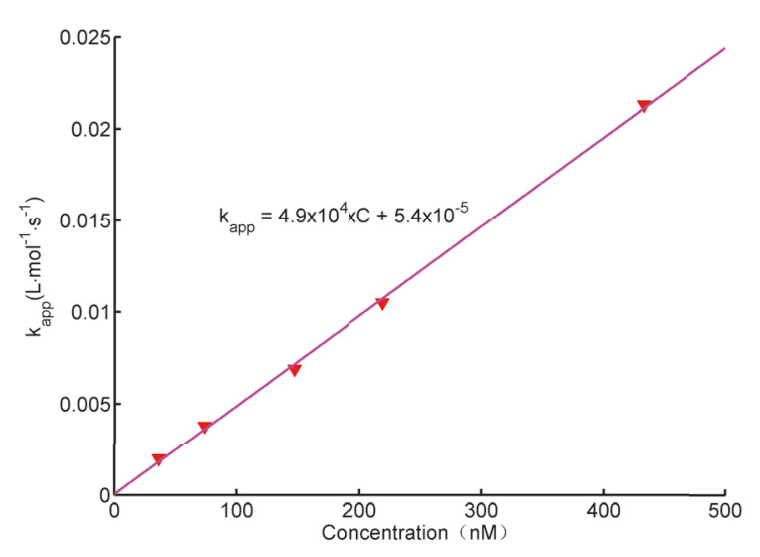

FIGURE 6: $\mathrm{k}_{\mathrm{app}}$ versus different concentrations of avidin.

to construct, so that many institutes or researchers can build their own 3D-SPR.

\section{Data Availability}

The data used to support the findings of this study are included within the article. 


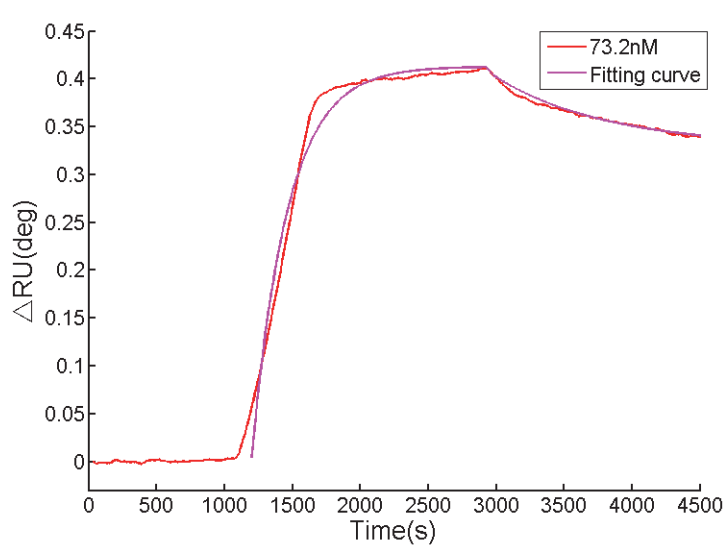

FIGURE 7: Interaction curve for 73.2nM avidin.

\section{Conflicts of Interest}

The authors declare that they have no conflicts of interest.

\section{Acknowledgments}

This work was supported by Beijing Natural Science Foundation (Z160002), the State Key Project of Fundamental Research (Grant 2014CB931900), UCAS Young Teacher Research Fund (Grants Y55103NY00, Y55103EY00, Y65201FY00, and Y25102TN00), and the Chinese Academy of Sciences Key Project Foundation (KFZD-SW-202).

\section{Supplementary Materials}

Figure S1 is mainly used to replace metal manufactured parts, including the optical base, holders, and fitting parts. $3 \mathrm{D}$ printing enables the rapid assembly of scattered devices such as laser, photodiode, prism, and Au-coated glass slide into a complete system. Figure S2 is the flow cell module which consists of rubber flow cell and stainless part. (Supplementary Materials)

\section{References}

[1] I. Stojanović, R. B. M. Schasfoort, and L. W. M. M. Terstappen, "Analysis of cell surface antigens by Surface plasmon resonance imaging," Biosensors \& Bioelectronics, vol. 52, pp. 36-43, 2014.

[2] K. M. Byun, N.-H. Kim, Y. H. Ko, and J. S. Yu, "Enhanced surface plasmon resonance detection of DNA hybridization based on ZnO nanorod arrays," Sensors and Actuators B: Chemical, vol. 155, no. 1, pp. 375-379, 2011.

[3] J. R. Wayment and J. M. Harris, "Biotin-avidin binding kinetics measured by single-molecule imaging," Analytical Chemistry, vol. 81, no. 1, pp. 336-342, 2009.

[4] T. Hiragun, Y. Yanase, K. Kose et al., "Surface plasmon resonance-biosensor detects the diversity of responses against epidermal growth factor in various carcinoma cell lines," Biosensors and Bioelectronics, vol. 32, no. 1, pp. 202-207, 2012.

[5] A. Kausaite, M. Van Dijk, J. Castrop et al., "Surface plasmon resonance label-free monitoring of antibody antigen interactions in real time," Biochemistry and Molecular Biology Education, vol. 35, no. 1, pp. 57-63, 2007.

[6] C. Wu, F. U. Rehman, J. Li et al., "Real-Time Evaluation of Live Cancer Cells by an in Situ Surface Plasmon Resonance and Electrochemical Study," ACS Applied Materials \& Interfaces, vol. 7, no. 44, pp. 24848-24854, 2015.

[7] Z. Zhu, M. Feng, L. Zuo et al., "An aptamer based surface plasmon resonance biosensor for the detection of ochratoxin $\mathrm{A}$ in wine and peanut oil," Biosensors and Bioelectronics, vol. 65, pp. 320-326, 2015.

[8] H. Vaisocherová, H. Šípová, I. Víšová et al., "Rapid and sensitive detection of multiple microRNAs in cell lysate by lowfouling surface plasmon resonance biosensor," Biosensors and Bioelectronics, vol. 70, pp. 226-231, 2015.

[9] F. Fernández, K. Hegnerová, M. Piliarik, F. Sanchez-Baeza, J. Homola, and M.-P. Marco, "A label-free and portable multichannel surface plasmon resonance immunosensor for on site analysis of antibiotics in milk samples," Biosensors and Bioelectronics, vol. 26, no. 4, pp. 1231-1238, 2010.

[10] X. Wang, S. Zhan, Z. Huang, and X. Hong, "Review: Advances and applications of surface plasmon resonance biosensing instrumentation," Instrumentation Science and Technology, vol. 41, no. 6, pp. 574-607, 2013.

[11] B. Liedberg, C. Nylander, and I. Lunström, "Surface plasmon resonance for gas detection and biosensing," Sensors and Actuators, vol. 4, pp. 299-304, 1983.

[12] S. Baumgarten and R. Robelek, "Surface plasmon resonance (SPR) sensors for the rapid, sensitive detection of the cellular response to osmotic stress," Sensors and Actuators B: Chemical, vol. 156, no. 2, pp. 798-804, 2011.

[13] Y. Liu, Q. Liu, S. Chen, F. Cheng, H. Wang, and W. Peng, "Surface plasmon resonance biosensor based on smart phone platforms," Scientific Reports, vol. 5, p. 12864, 2015.

[14] C.-W. Wu, C.-Y. Chiang, C.-H. Chen, C.-S. Chiang, C.-T. Wang, and L.-K. Chau, "Self-referencing fiber optic particle plasmon resonance sensing system for real-time biological monitoring," Talanta, vol. 146, pp. 291-298, 2016.

[15] D. Michel, F. Xiao, and K. Alameh, "A compact, flexible fiberoptic Surface Plasmon Resonance sensor with changeable sensor chips," Sensors and Actuators B: Chemical, vol. 246, pp. 258261, 2017.

[16] H. Guner, E. Ozgur, G. Kokturk et al., "A smartphone based surface plasmon resonance imaging (SPRi) platform for on-site biodetection," Sensors and Actuators B: Chemical, vol. 239, pp. 571-577, 2017.

[17] S. Dutta, K. Saikia, and P. Nath, "Smartphone based LSPR sensing platform for bio-conjugation detection and quantification," RSC Advances, vol. 6, no. 26, pp. 21871-21880, 2016.

[18] Z. Liu, Y. Wei, Y. Zhang et al., "A novel surface plasmon resonance sensor based on fiber butt-joint technology," Sensors and Actuators B: Chemical, vol. 221, pp. 1330-1334, 2015.

[19] M. George, K. R. Aroom, H. G. Hawes, B. S. Gill, and J. Love, "3D Printed Surgical Instruments: The Design and Fabrication Process," World Journal of Surgery, vol. 41, no. 1, pp. 314-319, 2017.

[20] G. Gaal, M. Mendes, T. P. de Almeida et al., "Simplified fabrication of integrated microfluidic devices using fused deposition modeling 3D printing," Sensors and Actuators B: Chemical, vol. 242, pp. 35-40, 2017.

[21] K. D. D. Willis, E. Brockmeyer, S. E. Hudson, and I. Poupyrev, "Printed optics:3D printing of embedded optical elements for 
interactive devices," in ACM Symposium on User Interface Software and Technology, 2012.

[22] T. L. Newcomb, A. M. Bruhn, B. Giles, H. M. Garcia, and N. Diawara, "Testing a Novel 3D Printed Radiographic Imaging Device for Use in Forensic Odontology," Journal of Forensic Sciences, vol. 62, no. 1, pp. 223-228, 2017.

[23] M. Sadia, A. Sośnicka, B. Arafat et al., "Adaptation of pharmaceutical excipients to FDM 3D printing for the fabrication of patient-tailored immediate release tablets," International Journal of Pharmaceutics, vol. 513, no. 1-2, pp. 659-668, 2016.

[24] A. Bonyár, H. Sántha, B. Ring, M. Varga, J. G. Kovács, and G. Harsányi, "3D Rapid Prototyping Technology (RPT) as a powerful tool in microfluidic development," Procedia Engineering, vol. 5, pp. 291-294, 2010.

[25] J. W. Zhao, Y. U. Hua Zhong, Y. Q. Wang, and Z. Liu, "Real time Characterization for Surface Wettibility of Cysteamine Self asembled Monolayers on Gold," Chemical Research in Chinese Universities, vol. 19, pp. 464-468, 1998.

[26] E. H. Williams, A. V. Davydov, A. Motayed et al., "Immobilization of streptavidin on $4 \mathrm{H}-\mathrm{SiC}$ for biosensor development," Applied Surface Science, vol. 258, no. 16, pp. 6056-6063, 2012.

[27] V. H. Pérez-Luna, M. J. O’Brien, K. A. Opperman et al., "Molecular recognition between genetically engineered streptavidin and surface-bound biotin," Journal of the American Chemical Society, vol. 121, no. 27, pp. 6469-6478, 1999.

[28] S. Zhao and W. M. Reichert, "Influence of Biotin Lipid Surface Density and Accessibility on Avidin Binding to the Tip of an Optical Fiber Sensor," Langmuir, vol. 8, no. 11, pp. 2785-2791, 1992. 

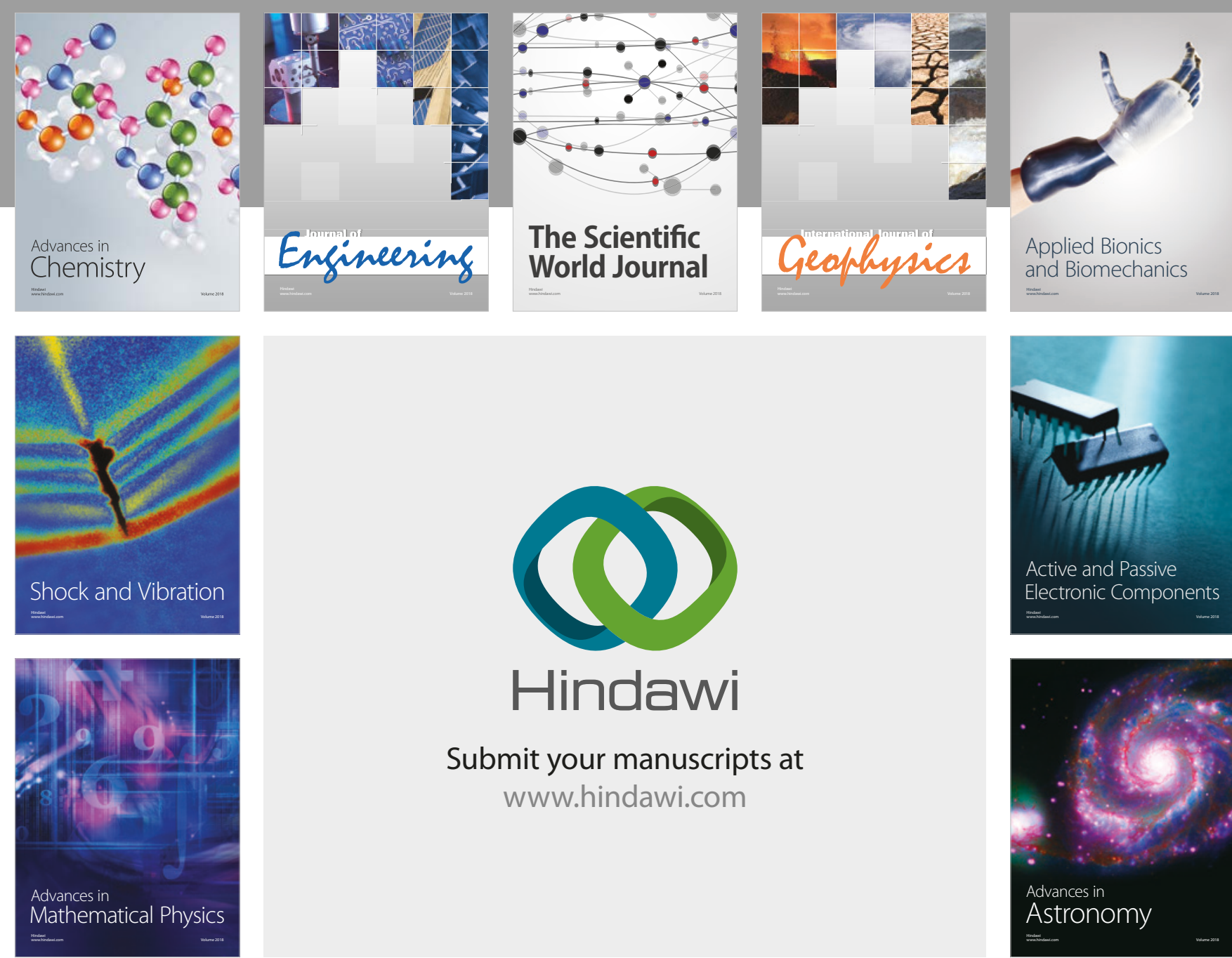

Submit your manuscripts at

www.hindawi.com

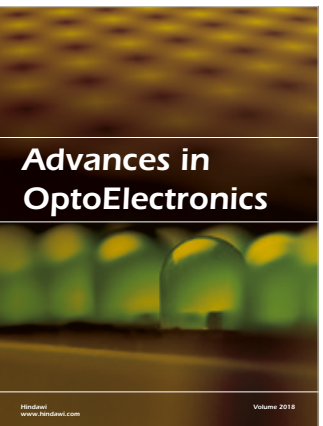

\section{Rotcting Machinery}
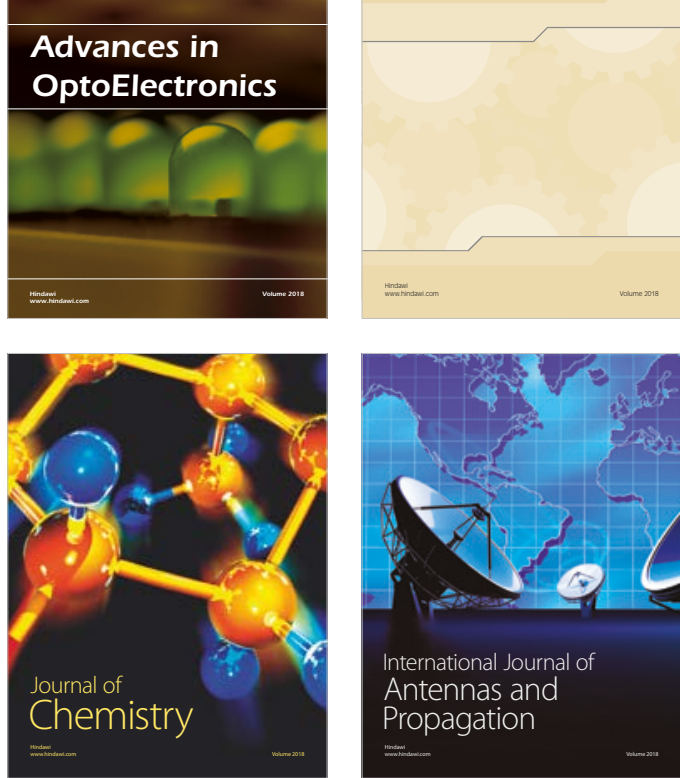

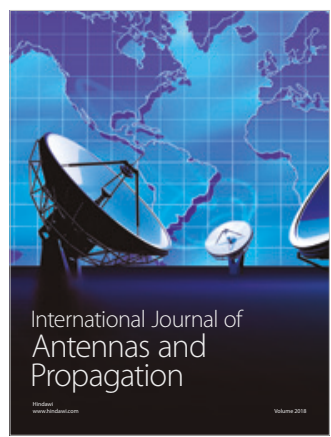

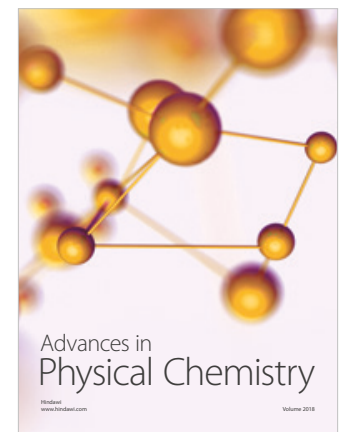

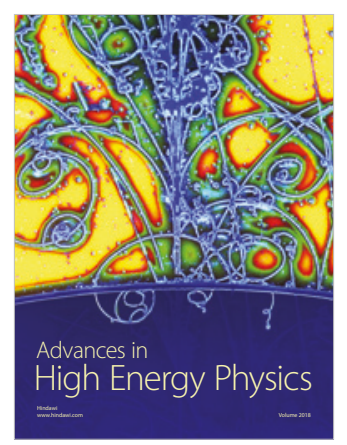

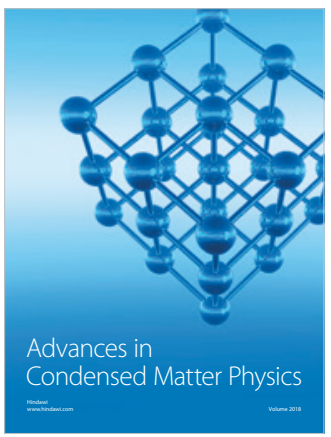

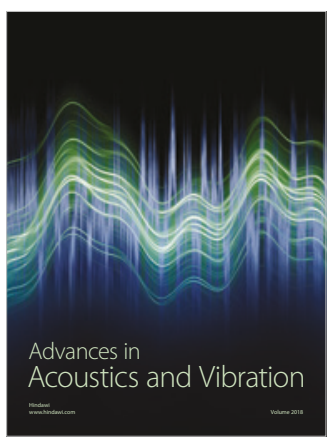

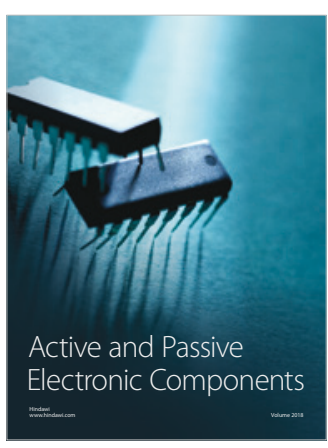
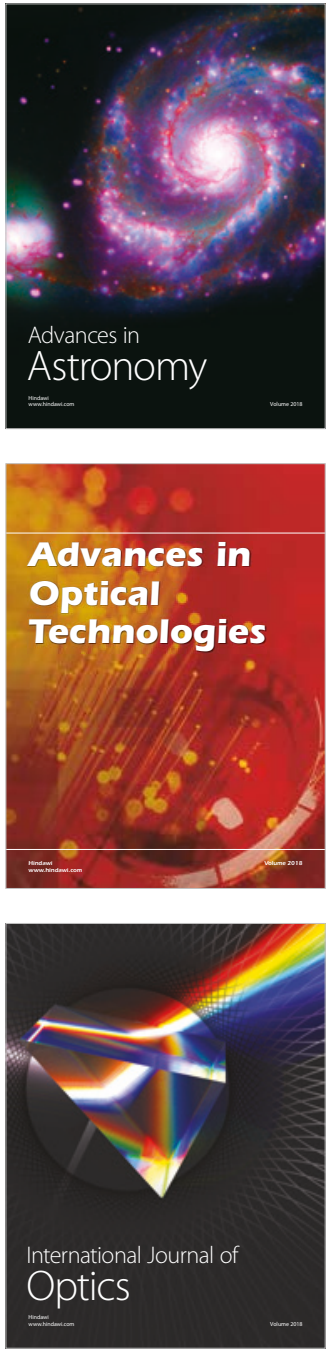